\title{
ON THE SPECTRUM OF LATTICE DIRAC OPERATORS
}

\author{
C. B. LANG \\ Institut für Theoretische Physik, Karl-Franzens-Universität Graz, \\ A-8010 Graz, AUSTRIA, \\ E-mail: christian.lang@kfunigraz.ac.at
}

\begin{abstract}
With the Schwinger model as example I discuss properties of lattice Dirac operators, with some emphasis on Monte Carlo results for topological charge, chiral fermions and eigenvalue spectra.
\end{abstract}

\section{Introduction}

\subsection{Continuum Concepts vs. Lattice Concepts}

Relativistic particle physics is described by relativistic quantum field theory; the theories explaining the fundamental interactions are gauge theories. QFT as it is formulated in terms of Lagrangians and functional integration has to be regularized. The only known regularization scheme that retains the gauge symmetry is replacement of the space-time continuum by a space-time lattice. For convenience and other reasons this is done in an Euclidean world.

The most prominent QFT is $\mathrm{QCD}$, the $\mathrm{SU}(3)$ gauge theory of quarks and gluons. There various non-perturbative phenomena appear intertwined: confinement and chiral symmetry breaking. The classical continuum gauge fields $A$ that are continuous and differentiable, living on compact manifolds, may be classified by a topological quantum number (the Pontryagin index) $Q(A)$. Changing through continuous deformations of the field from one such topologically defined sector to another is impossible.

Topology is closely related to the fermion zero modes; these are eigenstates (eigenvalue $E=0$ ) of the Dirac operator

$$
i \gamma_{\mu}\left(\partial_{\mu}-i e A_{\mu}\right) \psi=E \psi=0 \quad \rightarrow \quad \gamma_{5} \psi= \pm \psi .
$$

Due to the anti-commutation of $\gamma_{5}$ with $\mathcal{D}$ the zero modes can be chosen as eigenstates of $\gamma_{5}$ with definite chirality. The Atiyah-Singer index theorem (ASIT) 11 relates the topological charge $Q(A)$ of the background gauge field to these modes,

$$
Q(A)=\operatorname{index}(A) \equiv n_{+}-n_{-} .
$$

Talk at the Int. Conf. on Mathematical Physics and Stochastic Analysis (In honor of Ludwig Streit's 60th birthday), Lisbon, Oct. 1998 
where $n_{ \pm}$denotes the number of independent zero modes with positive or negative chirality, respectively. In two dimensions, another theorem of the continuum - the so-called Vanishing Theoreme - ensures that only either positive or negative chirality zero modes occur.

Quantization involves summation over non-differentiable fields and the lattice formulation does not provide for a unique definition of topological charge at all. Any lattice definition involves implicit or explicit assumptions, usually on the continuity and smoothness at the scale below one lattice spacing. Quantization usually means Monte Carlo integration on finite lattices and it is legitimate to question the ergodicity of the respective "simulation" with regard to the topological sectors.

Even the task of putting fermions on the lattice introduces limitations. Various lattice Dirac operators have been proposed. It has been demonstrated early 3 that within quite general assumptions (like locality and reflection positivity) it is not possible to have single chiral fermions; chiral symmetry has to be broken explicitly. For the simple Wilson action, the breaking of the symmetry is so bad that no trace of the chiral properties of the continuum theory is kept in the lattice theory. If, however, chiral symmetry is broken already on the level of the action, how can one hope to identify the (expected) spontaneous chiral symmetry breaking of the full theory? Is there a lattice version of the ASIT? As we will discuss below, recent developments do allow us to construct lattice Dirac operators, which break chiral symmetry in a minimal way.

Confinement, on the other hand, proved to be more straightforward. The gauge coupling $g$ enters the lattice gauge action in form of the multiplicative coupling $\beta=1 / g^{2}$. The lattice formulation works particularly well at strong coupling (small $\beta$ ), where confinement within a non-vanishing domain of the couplings was proved. In the Monte Carlo calculations for lattice QCD up to now no signal was found that indicates a phase transition between that confinement phase and the weak coupling (perturbative) regime; for finite temperature such a deconfinement transition was established.

In order to retrieve continuum QFT numbers for the physical quantities - like masses of hadronic bound states or certain matrix elements - one has to show that all dimensional physical quantities scale according to the scaling function of the lattice spacing $a(\beta)$, which in turn should asymptotically (in the continuum limit $\beta \rightarrow \infty, a \rightarrow 0)$ agree with continuum renormalization group scaling.

Current lattice studies have to live in that environment: Try to improve scaling properties in order to get reliable continuum results and try to deal with chirality without destroying it from the begin!

lisbon: submitted to World Scientific on September 5, 2018 


\subsection{Lattice Dirac Operators}

All Dirac operators have $\gamma_{5}$-hermiticity,

$$
\gamma_{5} \mathcal{D} \gamma_{5}=\mathcal{D}^{\dagger}
$$

It follows that

- The eigenvalues are either real or are complex conjugate pairs.

- The operator $\gamma_{5} \mathcal{D}$ has a real spectrum.

- We denote by $v_{i}$ the eigenvector of $\mathcal{D}$ for eigenvalue $\lambda_{i}$; then $\gamma_{5} v_{i}$ is an eigenvector of $\mathcal{D}^{\dagger}$ for the same eigenvalue.

- The diagonal entries of the chiral density matrix vanish for non-real eigenvalues: $\lambda_{i} \notin \operatorname{Re} \rightarrow\left\langle v_{i} \mid \gamma_{5} v_{i}\right\rangle=0$.

- The non-diagonal entries of the chiral density matrix vanish whenever their respective eigenvalues are not complex conjugate pairs: $\lambda_{i} \neq \bar{\lambda}_{j} \rightarrow$ $\left\langle v_{i} \mid \gamma_{5} v_{j}\right\rangle=0$.

An important conclusion is that only real eigenvalues lead to contributions to the diagonal elements. Thus these modes are the only candidates for zero modes in the continuum limit: in the continuum theory the (normalized) zero modes contribute to the diagonal entries, $\left\langle\psi \mid \gamma_{5} \psi\right\rangle= \pm 1$.

Chirality is necessarily broken for lattice Dirac operators. However, barely noticed for almost two decades, Ginsparg and Wilson $\exists$ formulated a condition (GWC) under which circumstances $\mathrm{g}$ remnants of the chiral symmetry survive in a lattice action for massless fermions. If the Dirac operator obeys

$$
\frac{1}{2}\left\{\gamma_{5}, \mathcal{D}\right\}=a \mathcal{D} \gamma_{5} R \mathcal{D}
$$

where $R$ denotes a local matrix, then chiral symmetry is violated only by a local term $\mathcal{O}(a)$. Dirac operators satisfying the GWC will be called GW Dirac operators.

Lüscher ${ }^{6}$ has pointed out the explicit form of the associated symmetry of the action,

$$
\psi \rightarrow \exp \left[i \theta \gamma_{5}(1-a R \mathcal{D})\right] \psi, \bar{\psi} \rightarrow \bar{\psi} \exp \left[i \theta(1-a \mathcal{D} R) \gamma_{5}\right] .
$$

The GWC provides a sensible way (respecting the Nielsen-Ninomiya theorem) to construct suitable Dirac operators. As has been shownd such actions cannot be ultra-local, but may be local, i.e. the coupling exponentially damped in real space. 


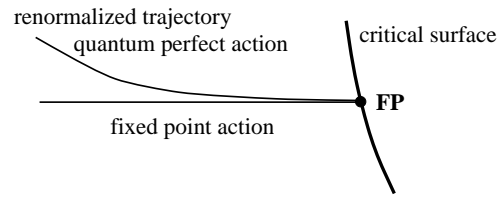

Figure 1. The FP action is the classical perfect action; the quantum perfect action may deviate from it away from the FP.

For certain classes of Dirac operators one may derive stringent bounds on the shape of the eigenvalue spectrum. This has been discussed in the framework of so-called fixed point (FP) actions 3 .

On the lattice real space renormalization group (RG) transformations are realized through block spin transformations. The field variables over a localized region around a site $x$ are averaged to produce the field variable of a coarser lattice. This mapping procedure in the space of configuration ensembles may be associated to a parameter flow in the space of actions. It is implicitly assumed that indeed a Gibbsian measure is suitable to describe the ensemble of blocked configurations.

The continuum limit of the quantum theory is obtained at a critical FP of the system (cf. Fig.11). The so-called renormalized trajectory in this space of actions leads to the FP and along its path there are no corrections to scaling. Ideally one could simulate the system at any point of the renormalized trajectory (with the so-called quantum perfect action) and obtain "perfect" continuum results. In reality that action may be quite complicated and has to be truncated in the number of coupling constants.

Hasenfratz et al.8 have suggested to determine instead the action at the FP of the RG transformation. For (asymptotically) free theories it may be determined from the classical field equations. The action has therefore been baptized "classical perfect action". Its classical predictions agree with those of the continuum action independent of the coarseness of the lattice. FP actions are solutions of the GWC 5 .

At the classical level, for FP actions, the Atiyah-Singer theorem finds correspondence on the lattice 5 ; at the quantum level, no fine tuning, mixing and current renormalization occur, and a natural definition for an order parameter of the spontaneous breaking of the chiral symmetry is possible 10 . $R$ is then local and bounded and as a consequence the spectrum of $\mathcal{D}$ in complex space is confined between two circles (cf. Fig.2),

$$
\left|\lambda-r_{\min }\right| \geq r_{\min }, \quad\left|\lambda-r_{\max }\right| \leq r_{\max },
$$




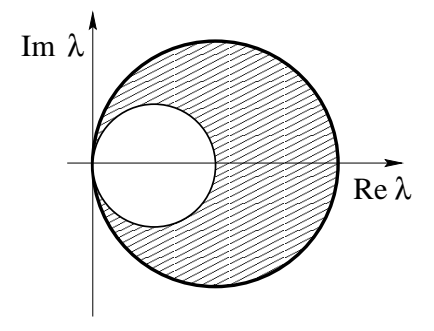

Figure 2. For FP Dirac operators the eigenvalues are confined in the shaded area of two circles tangential to the imaginary axis. The thick line indicates the position of the spectrum for $R=1 / 2$.

where the real numbers $r_{\min }$ and $r_{\max }$ are related to the maximum and minimum eigenvalue of $R$ respectively. For non-overlapping BSTs $R=1 / 2$ and (6) reduces to $|\lambda-1|=1$, i.e. the spectrum lies on a unit circle.

Independent implementations of the GWC are provided by the overlap formalism11, which allows the formulation of chiral fermions on the lattice. These solutions are obtained in an elegant way, as shown recently by Neuberger ${ }^{12}$, through some map of the Wilson operator with negative fermion mass. In this case we have $R=1 / 2$ and circular spectrum $|\lambda-1|=1$, too.

For $R=1 / 2$ the GWC assumes the simple form

$$
\mathcal{D}+\mathcal{D}^{\dagger}=\mathcal{D}^{\dagger} \mathcal{D}=\mathcal{D} \mathcal{D}^{\dagger}
$$

On the lattice, an index for $\mathcal{D}$ may be defined in a way analogous to the continuum, explicitly expressed by the relation

$$
\operatorname{index}(U)=-\operatorname{tr}\left(\gamma^{5} R \mathcal{D}\right) .
$$

For GW Dirac operators and $R=1 / 2$ this relation comes out trivially. Only the modes with real non-vanishing eigenvalues contribute to the trace in the r.h.s; since the overall chirality must be zero, it reproduces up to a sign index $(U)$. This index can be used to define a fermionic lattice topological charge, $Q_{\text {ferm }}(U) \equiv \operatorname{index}(U)$, for which the ASIT is satisfied by definition. For the FP action that fermionic definition coincides ${ }^{2}$ with the pure-gauge quantity $Q_{\mathrm{Fp}}(U)$, the FP topological charge 13 of the configuration $U$ :

$$
Q_{\text {ferm }}(U) \equiv \operatorname{index}(U)=Q_{\mathrm{Fp}}(U) .
$$

The non-obviousness of this relation relies on the fact that $Q_{\mathrm{Fp}}(U)$ can be defined in the pure gauge theory, without any regard to the fermion part. This result is particular for a FP action and has no counterpart for a general 
(non-FP) GW action. Of course, in practical implementations one relies on approximate parametrizations of the FP Dirac operator and the strictness of the relation is lost.

\subsection{Test Bed Schwinger Model}

As our test bed we consider a 2-dimensional (2D) quantum field theory with $\mathrm{U}(1)$ gauge group and $N_{f}$ flavors of fermions. The action for the massless continuum model reads

$$
S=\int d^{2} x\left(\frac{1}{4} F_{\mu \nu} F_{\mu \nu}+\sum_{f}^{N_{f}} \bar{\psi}_{f} \mathcal{D} \psi_{f}\right) .
$$

For $N_{f}=1$ this is the Schwinger model 4 .

This 2D version of $\mathrm{QED}$ resembles 4-dimensional $\mathrm{QCD}$ in various ways 15.16. Quarks are "trapped", i.e. in a mechanism superficially mimicking confinement we observe only bosonic asymptotic states. For $N_{f}=1$ this is the Schwinger boson (called $\eta$ by analogy to $4 \mathrm{D}$ ) with the mass $m_{\eta}=g / \pi$ for the physical gauge coupling $g$. For the 2 -flavor model one expects also a triplet of massless bosons (pions) 16 , although in 2D there can be not spontaneous symmetry breaking due to the Mermin-Wagner-Coleman theoremet 7 . Finally, there is a non-vanishing condensate $\langle\bar{\psi} \psi\rangle$ due to an anomaly.

In the lattice formulation the gauge action in the compact Wilsonformulation is written

$$
S_{g}=\beta \sum_{p \in \Lambda}\left(1-\operatorname{Re} U_{p}\right)
$$

where $\Lambda$ denotes the lattice $Z_{N}^{2}$ and the plaquette variable $U_{p}$ is the oriented product of links variables $U_{x, \mu} \in U(1)$ at site $x$ in direction $\mu=1,2$. In the continuum limit $U_{x, \mu} \simeq \exp \left(i a g A_{x, \mu}\right)$ where $A$ is the gauge field in the non-compact continuum formulation, $a$ denotes the lattice spacing, and $\beta=1 /\left(g^{2} a^{2}\right)$.

A lattice version for the integer geometric topological charge of the gauge field may be defined,

$$
Q(A)=\frac{g}{2 \pi} \int d^{2} x F_{12}(x) \quad \rightarrow \quad Q(U)=\frac{1}{2 \pi} \sum_{p} \operatorname{Im} \ln U_{p} .
$$

In torus geometry 18 (i.e. periodic boundary conditions) this number may be non-zero for compact gauge field configurations. 
The lattice fermion action is formally

$$
\bar{\psi} \mathcal{D}(m) \psi,
$$

where $\mathcal{D}(m)$ denotes the lattice Dirac operator matrix (fermion mass $m$ ) and the fermions are Grassmann fields. In a 2D context the Dirac matrices $\gamma_{\mu}$ and $\gamma_{5}$ are to be replaced by $\sigma_{\mu}$ and $\sigma_{3}$.

In the quantized theory the Grassmann integration over the fermions yields factors $(\operatorname{det} \mathcal{D})$ and the expectation value of some operator $C$ may be written

$$
\langle C\rangle=\frac{1}{Z} \int[d U] e^{S_{g}(U)}(\operatorname{det} \mathcal{D})^{N_{f}} C(U),
$$

where one usually samples over the gauge fields with some Monte Carlo procedure. In the so-called quenched approximation $\left(N_{f}=0\right)$ the fermionic determinant is not included; this essentially neglects the fermionic vacuum loops.

Dynamical fermions can be included either by incorporating them into the sampling probability measure for the gauge configurations or in the observable. The first case is realized in the so-called "Hybrid Monte Carlo" method. However, since the determinant may be negative, only even numbers of mass-degenerate fermions can be studied then. The second approach requires calculation of the determinant for each gauge configuration. It is plagued by the notorious sign problem, giving rise to possibly violent fluctuations and large statistical errors of the results.

The gauge integral should sample over all topological sectors. For Dirac operators which allow exact zero modes the determinant weight (for massless fermions) removes the corresponding contribution to the integral.

\section{Lattice Fermions and Topology}

We have been studying the lattice Schwinger model for various lattice sizes and values of the gauge coupling, both quenched and with dynamical fermions. Here we discuss some of our results, with some emphasis on the properties of the spectra of GW operators.

\subsection{The Wilson Dirac Operator}

The original Wilson action 19 for fermions has the form

$$
\mathcal{D}_{\mathrm{Wi}}=(m+2) \mathbf{1}-\frac{1}{2} M
$$




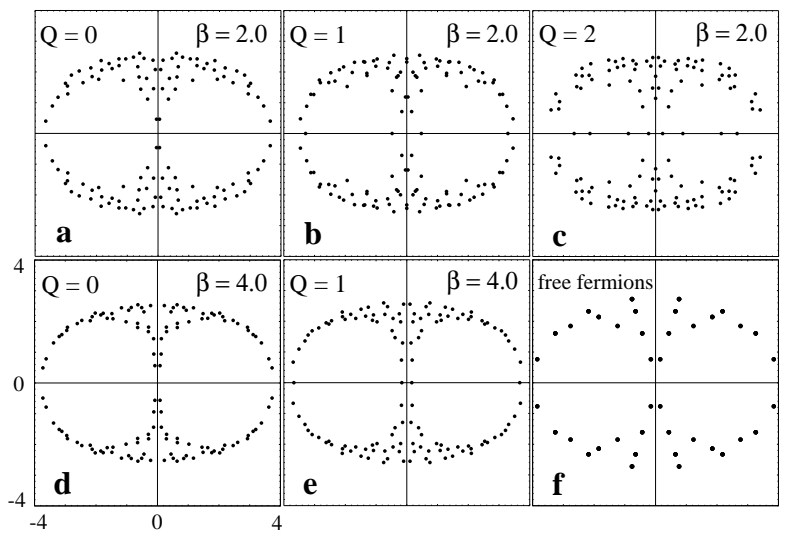

Figure 3. Spectrum of the hopping matrix $M$ for an $8 \times 8$ lattice at various values of $\beta$, close to $\kappa_{c}(\beta) ; Q$ denotes the (geometric definition of the) topological charge. The eigenvalues for free fermions are degenerate.

with the hopping matrix

$$
M_{x y}=\sum_{\mu=1,2}\left[\left(1+\sigma_{\mu}\right) U_{x y} \delta_{x, y-\mu}+\left(1-\sigma_{\mu}\right) U_{y x}^{\dagger} \delta_{x, y+\mu}\right] .
$$

The hopping parameter $\kappa$ defines the fermion mass, for free lattice fermions $\kappa=1 /(2 m+4)$. However, for the full theory with gauge interactions this relationship no longer holds. On one hand due to quark trapping there are no asymptotic fermions. On the other hand chiral symmetry is explicitly broken.

Since this breaking is a local feature, we expect that at some $\kappa_{c}(\beta)$ the chiral symmetry is formally restored in the sense, that the corresponding Ward identity is satisfied with a vanishing fermion mass parameter. This idea20 has been pursued in QCD21 and we have utilized it also in the Schwinger model with Wilson fermions in order to identify the position of the critical line, where the fermion mass parameter vanishes22.

In Fig. 3 we compare 23 typical eigenvalue spectra of the hopping matrix $M$ at different values of the gauge coupling and in different topological sectors. We find strong correlation between the number of real eigenvalues and the topological charge. Closer inspection leads to the conclusion, that indeed the real modes (counted according to their chirality 24 ) may be interpreted as the would-be zero modes. Actually one has to divide that number by a trivial multiplicity factor of 4 , since only the rightmost modes become zero modes of $\mathcal{D}_{\mathrm{Wi}}$, whereas the other ones are doublers at other corners of the Brillouin 
zone. The agreement of this number with the topological charge improves towards the continuum limit.

In that sense even the Wilson Dirac operator obeys the ASIT for $\beta \rightarrow$ $\infty$; e.g. at $\beta=4$ in $99 \%$ of the gauge configurations (sampled in a hybrid Monte Carlo simulation with dynamical fermions) the numbers of real modes (divided by 4) agrees with the topological charge. Also the Vanishing theorem is obeyed23.

\subsection{The Fixed Point Dirac Operator}

Although it was possible to obtain FP actions for some scalar or fermionic systems (cf. the summary in Ref.5) it turned out to be be a formidahle problem for realistic gauge-fermion systems like QCD. We have succeeded 22 to find explicitly an approximate FP action for the lattice Schwinger model. The FP Dirac operator is parametrized by a set of 429 terms bilinear in the fermion and anti-fermion fields,

$$
\mathcal{D}_{\mathrm{Fp}}=\sum_{i, x, s} \rho_{i}(f) \sigma_{i} U(x, f) \delta_{y, x+f} .
$$

The sum runs over paths $f$ connecting a central site $x$ with sites in a $7 \times 7$ neighborhood; $U(x, f)$ denotes the product of link gauge variables along that path. Standard symmetries are taken into account. Altogether we considered 123 independent coupling parameters.

The general idea is to start with a parametrization of the action, generate a set of gauge field configurations, perform the real space RG transformation and identify the blocked action. The matrix elements of the Dirac operator on the blocked configurations are then compared with the parametrization and the coupling constants adjusted. The whole procedure is iterated until the parameters converge.

We studied samples of 50 gauge configurations and $14 \times 14$ lattices at large values of $\beta$. We could show, that the resulting fermion action had couplings damped exponentially with their spatial extent; although the parametrized action is anyhow ultra-local by construction, this observation indicates that locality of an untruncated FP action seems feasible.

Using the FP action we determined the bosonic bound state propagators for the $N_{f}=1$ and 2 Schwinger model and found excellent rotational invariance and good scaling properties (cf. Fig.5), to be discussed below in context of other actions.

For the eigenvalue spectrum of the FP operator we found the situation shown in Fig. 4: The eigenvalues are distributed close to a unit circle, in particular towards the continuum limit (growing $\beta$ ). Towards the "hot" region the

lisbon: submitted to World Scientific on September 5, 2018 

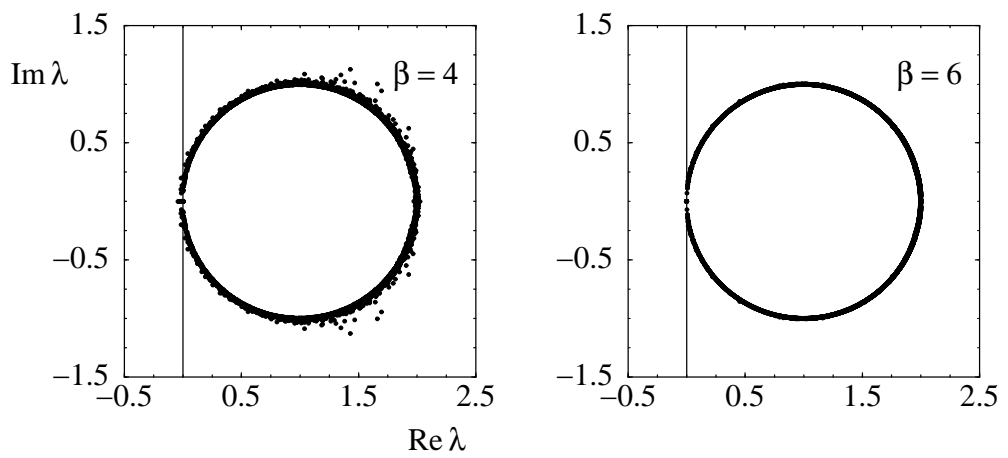

Figure 4. Eigenvalues for sets of 25 configurations (superimposed) for lattice size $16 \times 16$, sampled according to the compact gauge action (from Ref.26).

fuzziness increases. This demonstrates the predicted behavior for FP Dirac operators discussed in Sect. 1.2. The deviation from exact circularity is explained by the approximateness of the action, which gives raise to fluctuations at smaller $\beta$.

Most remarkable is, that indeed we do find (within the numerical accuracy) vanishing eigenvalues. These are individual zero modes and computing the matrix element $\left\langle v_{i} \mid \sigma_{3} v_{i}\right\rangle$ from their corresponding eigenvectors we find values \pm 1 . Thus these zero modes have definite chirality. The partner modes with opposite chirality are at the other edge of the spectrum, at $\lambda=2$, irrelevant in the continuum limit. The zero modes occur whenever the geometric topological charge of the gauge configuration is non-zero and its value agrees with the number of zero modes, even at finite $\beta$. This agreement with the ASIT is quantitatively much better than for the Wilson action.

\subsection{Neuberger's Overlap Dirac Operator}

Motivated by the overlap action 11 Neuberger proposed 12 to start with some Dirac operator with sufficiently negative mass, e.g. the Wilson operator at a value of $m$ corresponding to $\frac{1}{2 D}<\kappa<\frac{1}{2 D-2}$ and then construct

$$
\mathcal{D}_{\mathrm{Ne}}=\mathbf{1}+\gamma_{5} \epsilon\left(\gamma_{5} \mathcal{D}_{\mathrm{Wi}}\right) \text {. }
$$

Although the actual value of $\kappa$ used in this definition is largely arbitrary its choice may influence the approach to scaling in the continuum limit. The generalized sign function of the hermitian operator $\gamma_{5} \mathcal{D}_{\mathrm{Wi}}$ may be interpreted 

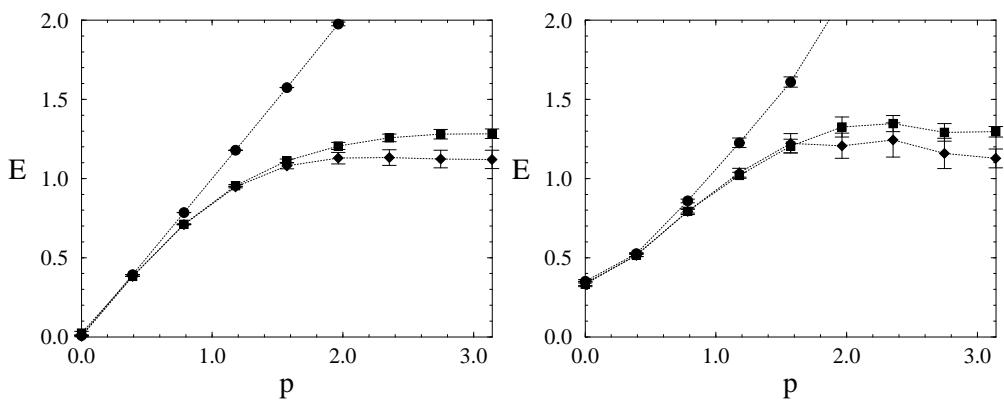

Figure 5. The dispersion relations $E(p)$ for the $\pi$ (left) and the $\eta$ (right) propagators, determined for three actions discussed in the text: Wilson (squares), fixed point (circles), Neuberger (diamonds) (from Ref.27).

as a realization of

$$
\frac{\mathcal{D}_{\mathrm{Wi}}}{\left|\mathcal{D}_{\mathrm{Wi}}\right|}=\frac{\mathcal{D}_{\mathrm{Wi}}}{\sqrt{\mathcal{D}_{\mathrm{Wi}}{ }^{\dagger} \mathcal{D}_{\mathrm{Wi}}}}=\gamma_{5} \frac{\gamma_{5} \mathcal{D}_{\mathrm{Wi}}}{\sqrt{\left(\gamma_{5} \mathcal{D}_{\mathrm{Wi}}\right)^{2}}}
$$

and is determined through the eigenvectors and the eigenvalues,

$$
\epsilon\left(\gamma_{5} \mathcal{D}_{\mathrm{Wi}}\right)=U \operatorname{Sign}(\Lambda) U^{\dagger} \quad \text { with } \gamma_{5} \mathcal{D}_{\mathrm{Wi}}=U \Lambda U^{\dagger} .
$$

$(\operatorname{Sign}(\Lambda)$ denotes the diagonal matrix of signs of the eigenvalue matrix $\Lambda$.)

Gauge configurations with non-zero topological charge imply 12 exact zero eigenvalues of $\mathcal{D}_{\mathrm{Ne}}$; therefore exact chiral modes are realizable. This was confirmed e.g. in Ref.27; we found an exactly circular eigenvalue spectrum and zero modes. Due to the freedom of choice of the hopping parameter in the Wilson action used to construct $\mathcal{D}_{\mathrm{Ne}}$ it cannot be excluded that some real doubler modes of the Wilson action are implicitly mistaken for zero modes leading to a violation of the ASIT at finite $\beta$. This situation can be efficiently improved, if one starts already with a better action, e.g. an approximate FP action.

An immediate question concerns locality of this operator. In 4D QCD it has been demonstrated 28 that for large enough $\beta$ one may expect locality and for a Monte Carlo generated ensemble of gauge configurations exponential falling off of the effective coupling parameters has been shown.

Neuberger's operator provides an explicit example of a GW action with optimal chirality properties. However, nothing can be concluded as to the scaling properties towards the continuum limit. In fact, in Fig. 目 we see, that the spectral properties of the bound states propagators are not improved over 
those of the original Wilson action. $\mathcal{D}_{\mathrm{Ne}}$ is automatically $\mathcal{O}(a)$ corrected 29.30 and thus improves scaling for the on-shell quantities; without at least introducing improvement of the current operators one would not expect improvement for the propagators, as shown by our results. The two features - chirality and scaling - seemingly may be considered quite separate issues. Optimal actions should allow chiral fermions and have good scaling properties.

\subsection{Spectral Distribution and chRMT}

The limiting value of the spectral density for small eigenvalues and large volume,

$$
-\pi \lim _{\lambda \rightarrow 0} \lim _{V \rightarrow \infty} \rho(\lambda)=\langle\bar{\psi} \psi\rangle
$$

provides an estimate for the chiral condensate due to the Banks-Casher

relation 31 . Exact zero modes are disregarded, only the density close to zero is of relevance.

In order to study this property of the lattice spectra (for the GW Dirac operators) one should map the eigenvalues from the circular shape to the imaginary axis. This is done by a stereographic projection

$$
\tilde{\lambda}=\frac{\lambda}{1-\frac{\lambda}{2}} \text {. }
$$

Near zero the resulting distribution on the (tangential) imaginary axis agrees with that on the circle. The generalization of such a projection for a general $\mathrm{GW}$ operator is the introduction of

$$
\tilde{\mathcal{D}}=\frac{\mathcal{D}}{1-R \mathcal{D}}
$$

which anti-commutes with $\gamma_{5}$, is anti-hermitian and has purely imaginary spectrum. A procedure to define a subtracted fermion condensate has been suggested by Hasenfratd (within the overlap formalism cf. Ref.32),

$$
\langle\bar{\psi} \psi\rangle_{\mathrm{sub}}=-\frac{1}{V}\left\langle\operatorname{tr}\left(\frac{1}{\mathcal{D}}-R\right)\right\rangle_{\text {gauge }}=-\frac{1}{V}\left\langle\operatorname{tr}\left(\frac{1}{\tilde{\mathcal{D}}}\right)\right\rangle_{\text {gauge }},
$$

(see also Ref.s 12,26,32,33). The expectation value includes the weight due to the fermionic determinant.

In Fig.6 (from Ref.27) the spectral density for the Neuberger operator is compared with that of the FP operator; near zero we find good agreement with each other and with the value expected from the continuum theory.

Studying the spectra of the Dirac operators suggests comparison with Random Matrix Theory 35 (RMT). The spectrum is separated in a fluctuation 


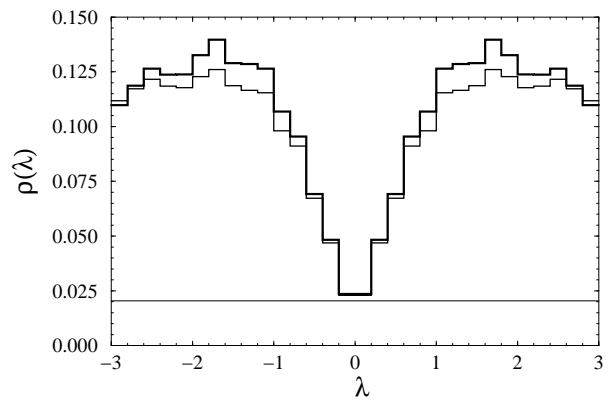

Figure 6. The unquenched $\left(N_{f}=1\right)$ eigenvalue density distribution $(16 \times 16, \beta=6)$ projected from the circle onto the imaginary axis for $\mathcal{D}_{\mathrm{Fp}}$ (thick lines) and $\mathcal{D}_{\mathrm{Ne}}$ (thin lines). The horizontal line denotes the continuum value at infinite volume.

part and a smooth background. The fluctuation part is conjectured to follow predictions in one of three universality classes. For chiral Dirac operators (chiral RMT36) these are denoted by chUE, chOE and chSE (chiral unitary, orthogonal or symplectic ensemble, respectively). Several observables have been studied in this theoretical context. Comparison of the data should verify the conjecture and allows one to determine the chiral condensate. This information is contained in both, the smooth average of the spectral distribution, and in the fluctuating part. In particular the distribution for the smallest eigenvalue $\rho_{\min }(\lambda)$ contains this observable: Its scaling properties with $V$ are given by unique functions of a scaling variable $z \equiv \lambda V \Sigma$, depending on the corresponding universality class. Usually this is the most reliable approach to determine $\Sigma$, which then serves as an estimate for the infinite volume value of the condensate in the chiral limit.

Within the Schwinger model we found 34 the universal properties of the (expected) chUE-class, unless the physical lattice volume is too small. In Fig. 7 we show the distribution of the smallest eigenvalue for two of the actions studied. Further examples, alsa including dynamical fermions and 4D applications, are discussed in Ref.s34.37.38. It has become evident, that chRMT describes the fluctuating part of the spectrum satisfactorily within the expected universality classes. It provides a means to separate the universal features from quantities like the chiral condensate, which have dynamic origin. Or, to say it (tongue in cheek) more provocative, to separate the known thus uninteresting, universal properties from the unknown, physical properties of the system. 

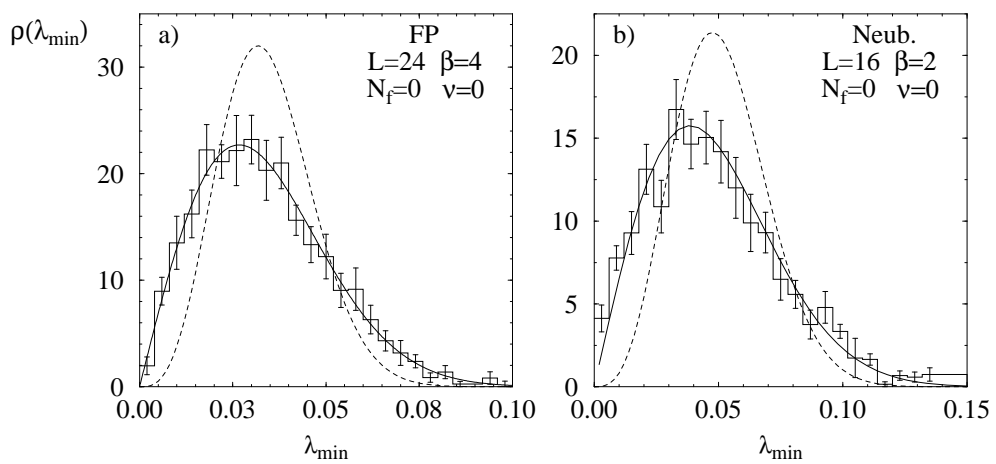

Figure 7. The distribution density for the smallest eigenvalue in the quenched $\left(N_{f}=0\right)$ case in the sector with topological charge zero. The full line gives the prediction for the chUE class, the broken line would correspond to the chSE universality class.

\section{Conclusion}

The main messages I wanted to deliver here are

- Lattice gauge theory has made a big step forward in the understanding of chiral fermions and their lattice formulation. The Ginsparg-Wilson relation is a cornerstone in that development. Meanwhile $\mathrm{U}(1)$ chiral gauge theories with anomaly-free multiplets of Weyl fermions have been constructed properly 39 .

- Neuberger's overlap action is a wonderful testing ground for chirality; its implementation in $4 \mathrm{D}$ is computationally expensive. Perfect actions ideally provide the best of both, chirality and scaling, but their construction in realistic $4 \mathrm{D}$ models seems to have unsurmountable problems. In a $2 \mathrm{D}$ gauge theory, however, we were able to construct an explicit example, which has all the beautiful features expected.

- The spectra of Dirac operators have universal features, which are described by chiral RMT. This opens the path to efficient methods to extract from the data non-perturbative quantities like the chiral condensate.

- The Schwinger model has provided an excellent test case to study various features also observed in QCD. Although maybe of different dynamical origin, mechanisms like confinement, chirality and symmetry breaking can be nicely studied in their lattice realization. This may lead to a better understanding also for the $4 \mathrm{D}$ theories. 


\section{Acknowledgments}

Ludwig Streit has been a colleague and friend since long; I learned a lot from him although we never collaborated (except for surfing in Santa Barbara). I want to thank F. Farchioni, C. Gattringer, I. Hip, T. Pany and M. Wohlgenannt for collaboration on various topics within the Schwinger model and for allowing me to present also some of their contributions in this text. This write-up tries to present the results available at the conference in Lisbon, Oct. 1998; only the references have been brought up to date. Support by Fonds zur Förderung der Wissenschaftlichen Forschung in Österreich, Project P11502-PHY, is gratefully acknowledged.

\section{References}

1. M. Atiyah and I. M. Singer, Ann. Math. 93, 139 (1971).

2. J. Kiskis, Phys. Rev. D 15, 2329 (1977); N. K. Nielsen and B. Schroer, Nucl. Phys. B 127, 493 (1977); M. M. Ansourian, Phys. Lett. 70B, 301 (1977).

3. H. Nielsen and M. Ninomiya, Nucl. Phys. B 185, 20 (1981); ibid. 193, 173 (1981); ibid. 195, 541 (1982).

4. P. H. Ginsparg and K. G. Wilson, Phys. Rev. D 25, 2649 (1982).

5. P. Hasenfratz, Nucl. Phys. B (Proc. Suppl.) 63A-C, 53 (1998).

6. M. Lüscher, Phys. Lett. B 428, 342 (1998).

7. I. Horvath, Phys. Rev. Lett. 81, 4063 (1998).

8. P. Hasenfratz and F. Niedermayer, Nucl. Phys. B 414, 785 (1994).

9. P. Hasenfratz, V. Laliena, and F. Niedermayer, Phys. Lett. B 427, 125 (1998).

10. P. Hasenfratz, Nucl. Phys. B 525, 401 (1998).

11. R. Narayanan and H. Neuberger, Phys. Lett. B 302, 62 (1993); Phys. Rev. Lett. 71, 3251 (1993); Nucl. Phys. B 412, 574 (1994); ibid. 443, 305 (1995).

12. H. Neuberger, Phys. Lett. B 417, 141 (1998); ibid. 427, 353 (1998).

13. M. Blatter, R. Burkhalter, P. Hasenfratz, and F. Niedermayer, Phys. Rev. D 53, 923 (1996).

14. J. Schwinger, Phys. Rev. 125, 397 (1962); ibid. 128, 2425 (1962).

15. J. H. Lowenstein and J. A. Swieca, Ann. Phys. 68, 172 (1971); S. Coleman, R. Jackiw, and L. Susskind, Ann. Phys. 93, 267 (1975); S. Coleman, Ann. Phys. 101, 239 (1976); J. Fröhlich and E. Seiler, Helv. Physica Acta 49, 889 (1976); L. V. Belvedere, J. A. Swieca, K. D. Rothe, and B. Schroer, Nucl. Phys. B 153, 112 (1979); J. Challifour and D. 
Weingarten, Ann. Phys. (N.Y.) 123, 61 (1979); H. Joos and S. I. Azakov, Helv. Phys. Acta 67, 723 (1994); H. Dilger, Nucl. Phys. B 434, 321 (1995); H. Dilger and H. Joos, Nucl. Phys. B (Proc. Suppl.) 34, 195 (1994).

16. C. R. Gattringer and E. Seiler, Ann. Phys. 233, 97 (1994).

17. N. D. Mermin and H. Wagner, Phys. Rev. Lett. 17, 1133 (1966); S. Coleman, Commun. Math. Phys. 31, 259 (1973).

18. H. Joos, Helv. Phys. Acta 63, 670 (1990); I. Sachs and A. Wipf, Helv. Phys. Acta 65, 653 (1992).

19. K. G. Wilson, Phys. Rev. D 10, 2445 (1974).

20. M. Bochicchio et al., Nucl. Phys. B 262, 331 (1985).

21. K. Jansen et al., Phys. Lett. B 372, 275 (1996).

22. I. Hip, C. B. Lang, and R. Teppner, Nucl. Phys. (Proc. Suppl.) 63, 682 (1998).

23. C. R. Gattringer, I. Hip, and C. B. Lang, Nucl. Phys. B 508, 329 (1997).

24. P. Hernández, Nucl. Phys. B 536, 345 (1998).

25. C. B. Lang and T. K. Pany, Nucl. Phys. B 513, 645 (1998); Nucl. Phys. B (Proc. Suppl.) 63A-C, 898 (1998).

26. F. Farchioni, C. B. Lang, and M. Wohlgenannt, Phys. Lett. B 433, 377 (1998).

27. F. Farchioni, I. Hip, and C. B. Lang, Phys. Lett. B 443, 214 (1998).

28. P. Hernández, K. Jansen, and M. Lüscher, hep-lat/9808010, CERNTH/98-250 (unpublished).

29. Y. Kikukawa, R. Narayanan, and H. Neuberger, Phys. Lett. B 399, 105 (1997).

30. F. Niedermayer, Nucl. Phys. B (Proc.Suppl.) 73, 105 (1999).

31. T. Banks and A. Casher, Nucl. Phys. 169, 103 (1980).

32. H. Neuberger, Phys. Rev. D 57, 5417 (1998).

33. T.-W. Chiu and S. V. Zenkin, Phys. Rev. D 59, 074501 (1999).

34. F. Farchioni, I. Hip, C. B. Lang, and M. Wohlgenannt, Nucl. Phys. B 549, 364 (1999).

35. T. Guhr, A. Müller-Groeling, and H. A. Weidenmüller, Phys. Rep. 299, 189 (1998).

36. H. Leutwyler and A. Smilga, Phys. Rev. D 46, 5607 (1992); E. V. Shuryak and J. J. M. Verbaarschot, Nucl. Phys. A 560, 306 (1993); J. J. M. Verbaarschot, Phys. Rev. Lett. 72, 2531 (1994); P. H. Damgaard, Phys. Lett. B 424, 322 (1998); P. H. Damgaard, J. C. Osborn, D. Toublan, and J. J. M. Verbaarschoot, Nucl. Phys. B 547, 305 (1999); G. Akemann and P. H. Damgaard, Nucl. Phys. B 528, 411 (1998); J. C. Osborn, D. Toublan, and J. J. M. Verbaarschoot, Nucl. 
Phys. B 540, 317 (1999).

37. M. E. Berbenni-Bitsch et al., Phys. Rev. Lett. 80, 1146 (1998); M. E. Berbenni-Bitsch, S. Meyer, and T. Wettig, Phys. Rev. D 58, 071502 (1998); M. E. Berbenni-Bitsch et al., Nucl. Phys. B (Proc. Suppl.) 73, 605 (1999).

38. R. G. Edwards, U. M. Heller, J. Kiskis and R. Narayanan, Phys. Rev. Lett. 82 (1999) 4188.

39. M. Lüscher, Nucl. Phys. B 549, 295 (1999); and hep-lat/9904009 (unpublished). 